\title{
CircRNA_100290 Promotes GC Cell Proliferation And Invasion Via miR-29b-3p/ITGA11 Axis And Is Regulated By EIF4A3
}

\section{Gang Wang}

Shandong Qianfoshan Hospital https://orcid.org/0000-0001-9318-6251

\section{Dan Sun}

The First Affiliated Hospital of China Medical University

\section{Wenhui Li}

The First Affiliated Hospital of China Medical University

\section{Yan Xin ( $\nabla$ yxin@cmu.edu.cn )}

Laboratory of Gastrointestinal Onco-Pathology, Cancer Institute, The First Affiliated Hospital of China Medical University. 155 Nanjing North Street, Heping District, Shenyang, Liaoning Province, China. https://orcid.org/0000-0002-8809-5803

\section{Primary research}

Keywords: circRNA_100290, miR-29b-3p/ITGA11 axis, EIF4A3, EMT, gastric cancer

Posted Date: January 14th, 2021

DOl: https://doi.org/10.21203/rs.3.rs-143764/v1

License: (c) (1) This work is licensed under a Creative Commons Attribution 4.0 International License. Read Full License 


\section{Abstract}

Background: Circular RNA (circRNA) has been reported as an important regulator in the development and progression of various carcinomas. However, the role of circRNA_100290 in gastric cancer (GC) is still unclear. This study aimed to investigate the role of circRNA_100290 in GC invasion and metastasis and its possible mechanism.

Methods: The expression of circRNA_100290 in GC cells and tissues were examined using quantitative real-time polymerase chain reaction (qRT-PCR). The role of circRNA_100290 in cell proliferation, migration, and invasion was evaluated on AGS and HGC-27 cell lines in vitro. Bioinformatics tools, dualluciferase reporter assay, Western blot assay and qRT-PCR were used to explore the downstream pathways of circRNA_100290. The mechanism underlying the regulation of the expression of circRNA_100290 was explored using RNA immunoprecipitation, qRT-PCR, and Western blot assays.

Results: The expression of circRNA_100290 was found significantly upregulated in GC cells and 102 GC tissues, high expression of circRNA_100290 in GC was closely related to Borrmann's types, lymph node metastasis and tumor-node-metastasis staging. In vitro, knockdown of circRNA_100290 in AGS and HGC27 cells significantly inhibited cell proliferation, migration, and invasion. Mechanistically, dual-luciferase reporter assay confirmed a direct binding between circRNA_100290 and miR-29b-3p, which targets ITGA11, an oncogene which is closely related to epithelial-mesenchymal transition (EMT). In addition, EIF4A3, one of RNA binding proteins (RBPs), could inhibit the formation of circRNA_100290 via enriching flanking sites of circRNA_100290. Low expression of EIF4A3 in GC was related to a worse prognosis.

Conclusions: Elevated circRNA_100290 in GC promotes cell proliferation, invasion and EMT via miR-29b3p/ITGA11 axi and might be regulated by EIF4A3. CircRNA_100290 might be a promising biomarker and target for GC therapy.

\section{Full Text}

This preprint is available for download as a PDF.

\section{Figures}




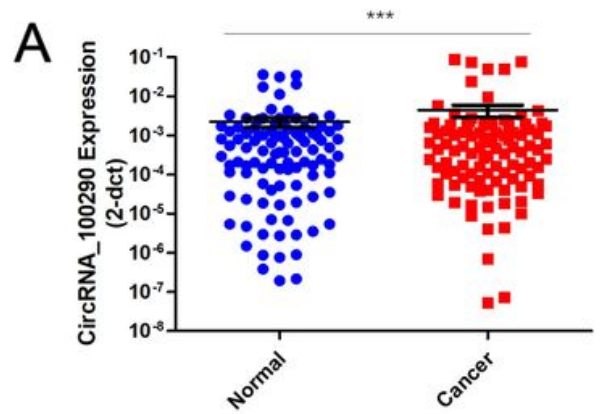

C

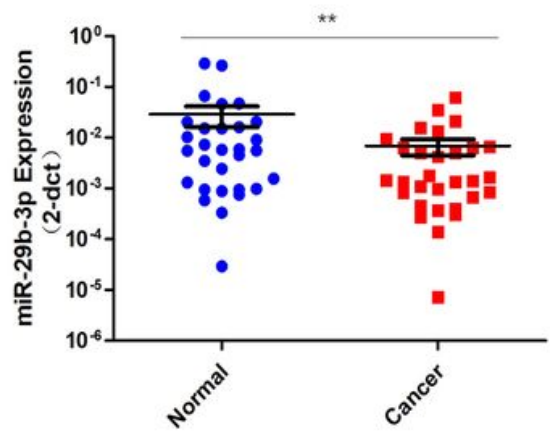

$\mathrm{F}$
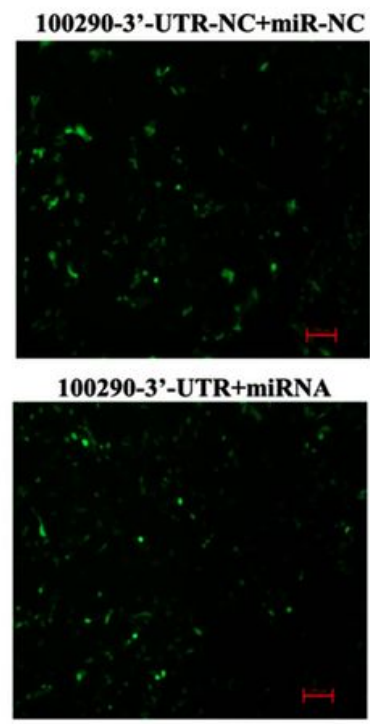

B

\begin{tabular}{|c|c|c|c|c|}
\hline miR Name $\Rightarrow$ & transcript nam & $\begin{array}{l}\text { leftmost position of } \\
\text { predicted target site }\end{array}$ & $\begin{array}{l}\text { folding energy } \\
\text { (in-Kcal/mol) }\end{array}$ & heteroduplex \\
\hline hsa-miR-29b-3p & circRNA_100290 & 401 & -13.00 & $\begin{array}{l}\text { AGCCATGAAGTGAAACATGGTGCTO } \\
\text { |:| ||| | || |||||||| } \\
\text { TTGTGACTAAAGTT--TRCCACGAT }\end{array}$ \\
\hline
\end{tabular}

D

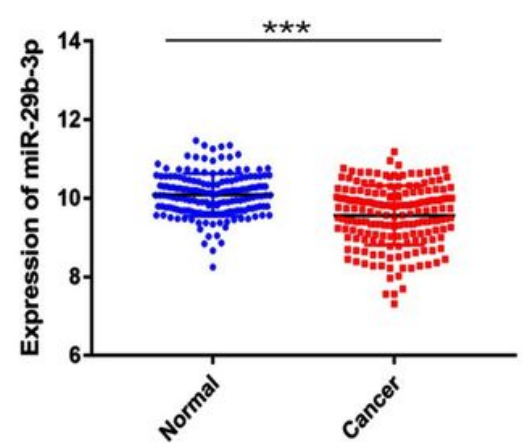

$\mathrm{E}$

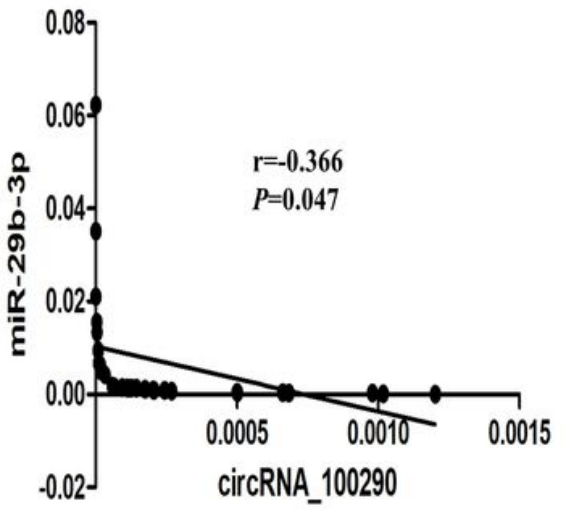

G

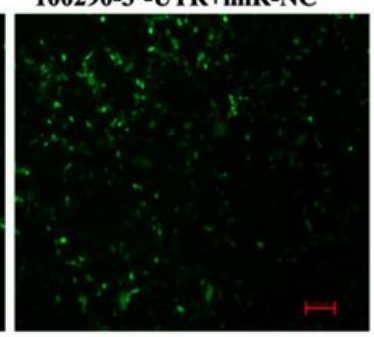

100290-3'-UTR-MU+miRNA
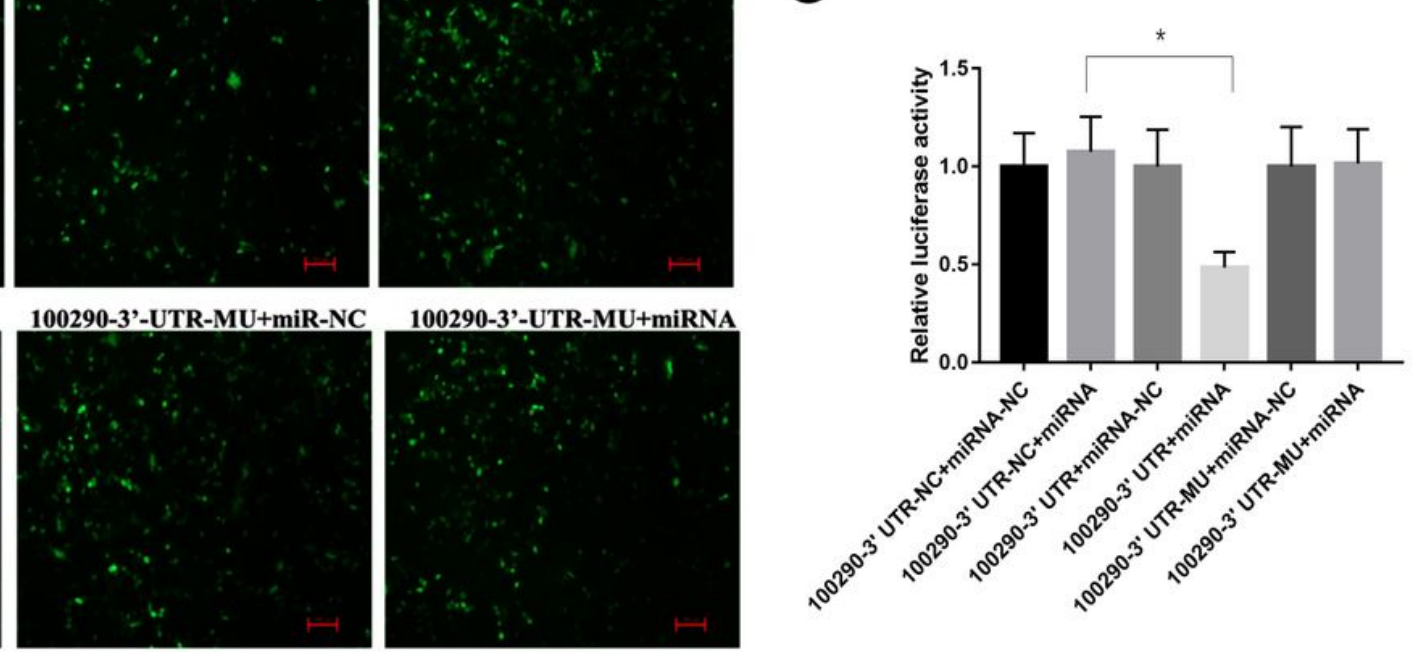

\section{Figure 1}

The expression pattern and correlation of circRNA_100290 and hsa-miR-29b-3p in human GC. (A) CircRNA_100290 expression in 102 GC and paired normal tissues was detected by qRT-PCR. Data are means \pm SEM. ${ }^{\star \star \star *} P<0.0001$. (B) RNA22 V2.0 was used to predict the interaction between circRNA_100290 and hsa-miR-29b-3p. (C) The expression of miR-29b-3p in 31 GC and matched normal mucosa was evaluated by qRT-PCR. Data are means \pm SEM. $* * P<0.01$. (D) Expression analysis of miR29b-3p in 184 GC tissues and 168 normal tissues was performed according to EBI database. ${ }^{* \star *} \mathrm{P}<0.001$. (E) Negative correlation between circRNA_100290 and miR-29b-3p expressions was observed by Pearson 
correlation analysis. $r=-0.366, P=0.047$. $(F)$ Evaluation of transfection efficiency of luciferase reporter gene plasmid by fluorescence microscopy. (G) Relative of luciferase activity of each group was calculated. Luciferase activity of the control group was normalized to $1,{ }^{\star} \mathrm{P}<0.05$.

A

\begin{tabular}{|c|c|c|c|c|c|c|c|c|c|}
\hline name & geneName & position & targetScanSites & picTarSites & RNA22Sites & PITASites & miRandaSites & programNum & CancerNum \\
\hline $\begin{array}{l}\frac{\text { hsa- }}{\text { miR- }} \\
\underline{2 g b-}- \\
\underline{3 p} p\end{array}$ & $\underline{\text { ITGA11 }}$ & $\begin{array}{l}\text { chr15:68595071- } \\
68595077[-]\end{array}$ & $65[1]$ & $65[1]$ & $0[0]$ & $0[0]$ & $0[0]$ & 2 & 7 \\
\hline
\end{tabular}

B

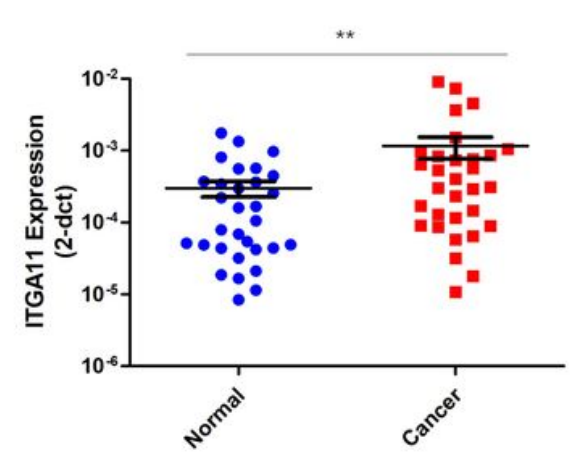

$E$

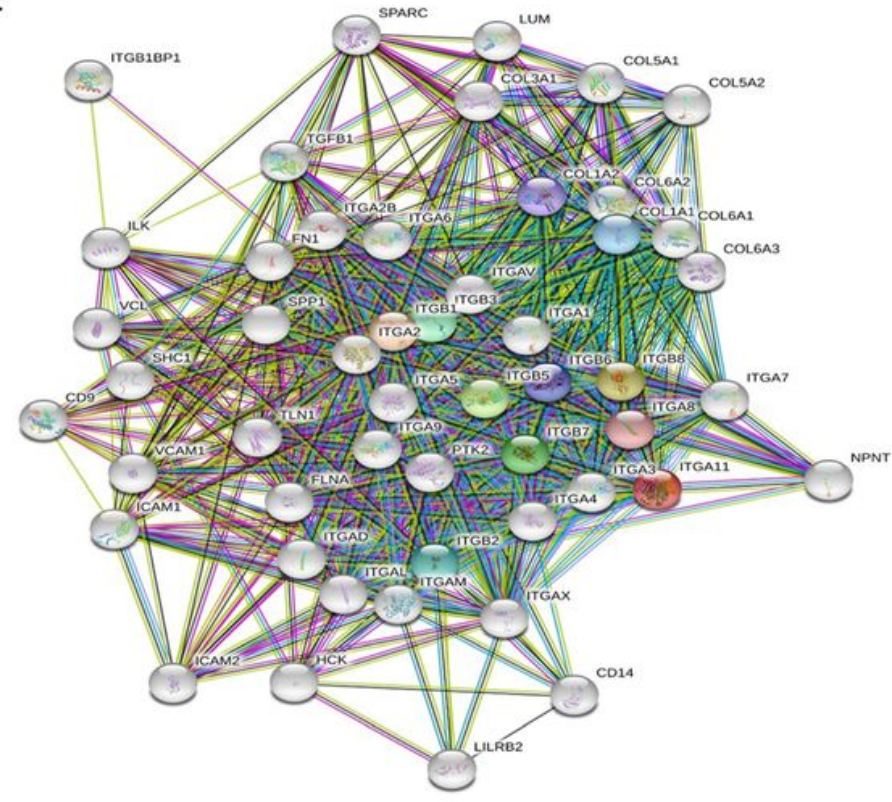

$\mathrm{C}$

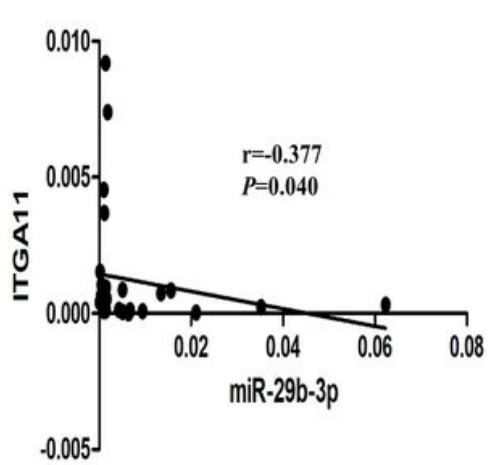

Known Interactions

E-O from curated databases

e-O experimentally determined

C-9 gene neighborhood

$\mathrm{A}-\mathrm{O}$ gene fusions

- gene co-occurrence

Biological Process $(00)$

\begin{tabular}{|c|c|c|c|}
\hline 60:term & description & \multicolumn{2}{|c|}{ countingeneset falsediscoveryate } \\
\hline 60:0030198 & extracelluluar matitix organizzation & $410+296$ & $8.539-61$ \\
\hline $60: 007229$ & integerin-mediated signalingpathway & 270084 & $1.03 e-45$ \\
\hline 60:0007155 & cellathesion & 4106843 & $1.188+43$ \\
\hline 60:0007160 & cellnatixix adhesion & $240: 119$ & $4.73 e-36$ \\
\hline 60:0031589 & Cel|substatide adhesion & 2500162 & $3.599-35$ \\
\hline 60:0007160 & cell surfacer receptor signaling pathway & 4002198 & $5.160-26$ \\
\hline 60:00164677 & cell migration & 2908812 & 20.77e-24 \\
\hline
\end{tabular}

\section{Figure 2}

The correlation of hsa-miR-29b-3p and ITGA11 in human GC. (A) One of the most possible targeted genes of hsa-miR-29b-3p was predicted by Starbase. The result had shown that ITGA11 had binding sites with miR-29b-3p. (B) ITGA11 expression in $31 \mathrm{GC}$ and paired normal tissues was detected by qRT-PCR. $\star \star P<0.01$. (C) Negative correlation between miR-29b-3p and ITGA11 expressions was observed by Pearson correlation analysis. $r=-0.317, P=0.009$. (D) Prognostic value of the expression of ITGA11 in 354 
patients with GC in the TCGA database. (E) protein-protein interaction analysis and gene ontology analysis of ITGA11 using STRING software.

A
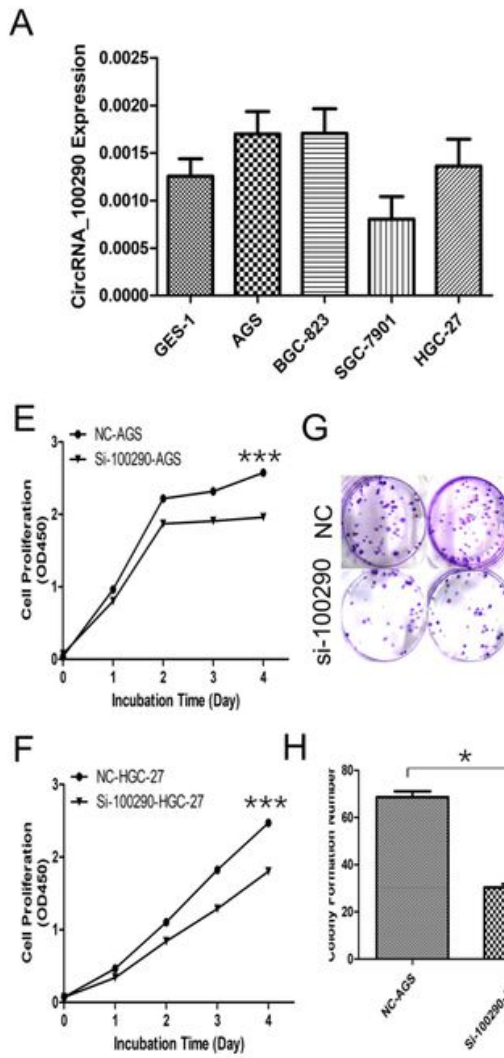

B

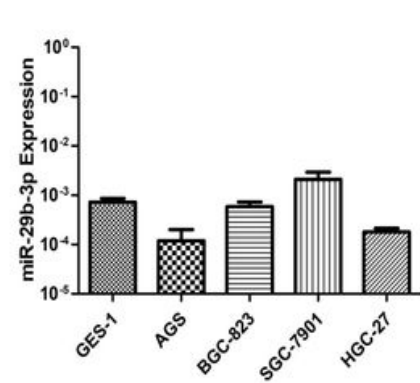

C

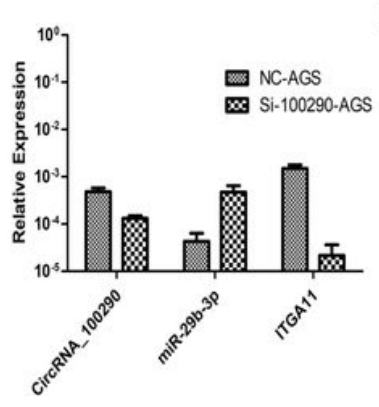

K

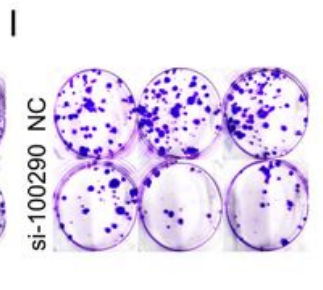

$J$

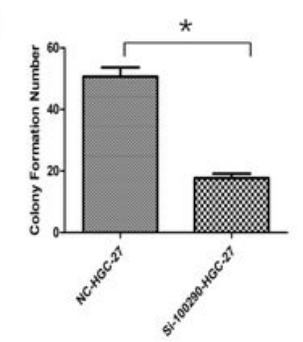

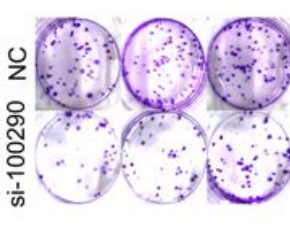

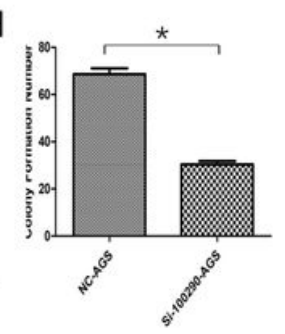

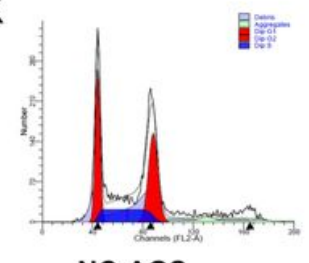

NC-AGS

M

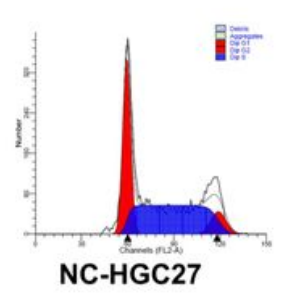

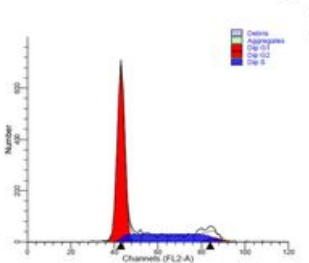

si-100290_AGS

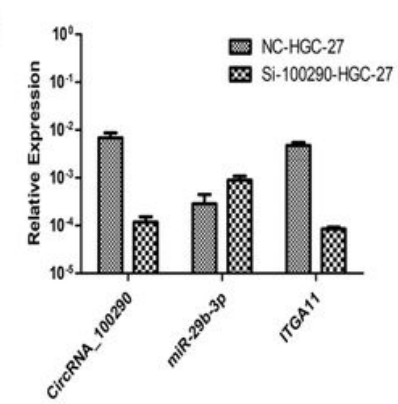

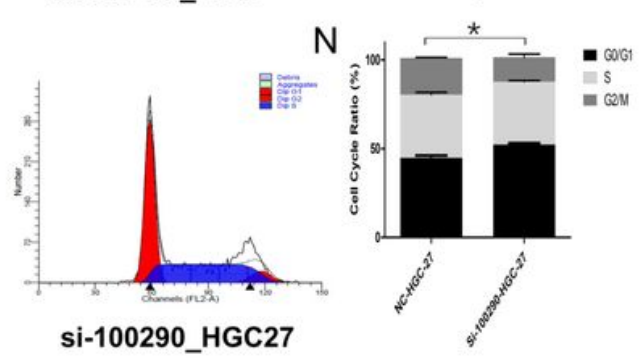

\section{Figure 3}

Knocking down circRNA_100290 inhibits GC cell viability, colony-formation and induces G0/G1 cycle arrested. (A) Expressions of circRNA_100290 was detected in four GC cell lines AGS, BGC823, SGC7901, HGC27 and human immortalized normal gastric epithelial cells GES-1 by qRT-PCR assay. (B) Expressions of miR-29b-3p was detected in AGS, BGC823, SGC7901, HGC27 and GES-1 cell lines by qRT-PCR assay. (C) Detection of miR-29b-3p and ITGA11 expression in AGS cell line by qRT-PCR assay after circRNA_100290 siRNA transfection was conducted. (D) Detection of miR-29b-3p and ITGA11 expression in HGC-27 cell line by qRT-PCR assay after circRNA_100290 siRNA transfection was conducted. (E\&F) CCK-8 assays were used to evaluate the effects of 22 circRNA_100290 on the proliferation abilities of AGS and HGC27 cells. Knocking-down circRNA_100290 expression significantly inhibited GC cells viability. ${ }^{* * *} P<0.001$. (G\&I) Colony formation assays were performed to evaluate the effects of circRNA_100290 on colony-formation abilities of AGS and HGC27 cells. (H\&J) The statistical graphs showed that decreasing circRNA_100290 expression suppressed the AGS and HGC27 cells colonyformation ability $\left({ }^{*} P<0.05\right)$. (K\&M) The flow cytometry was used to analyze the effects of circRNA_100290 on AGS and HGC27 cell cycle. (L\&N) The statistical graphs demonstrated that decreasing circRNA_100290 expression increased the percentage of G0/G1 phase in AGS and HGC27 cells compared with $\mathrm{NC}$ control groups $\left({ }^{*} \mathrm{P}<0.05\right)$. 


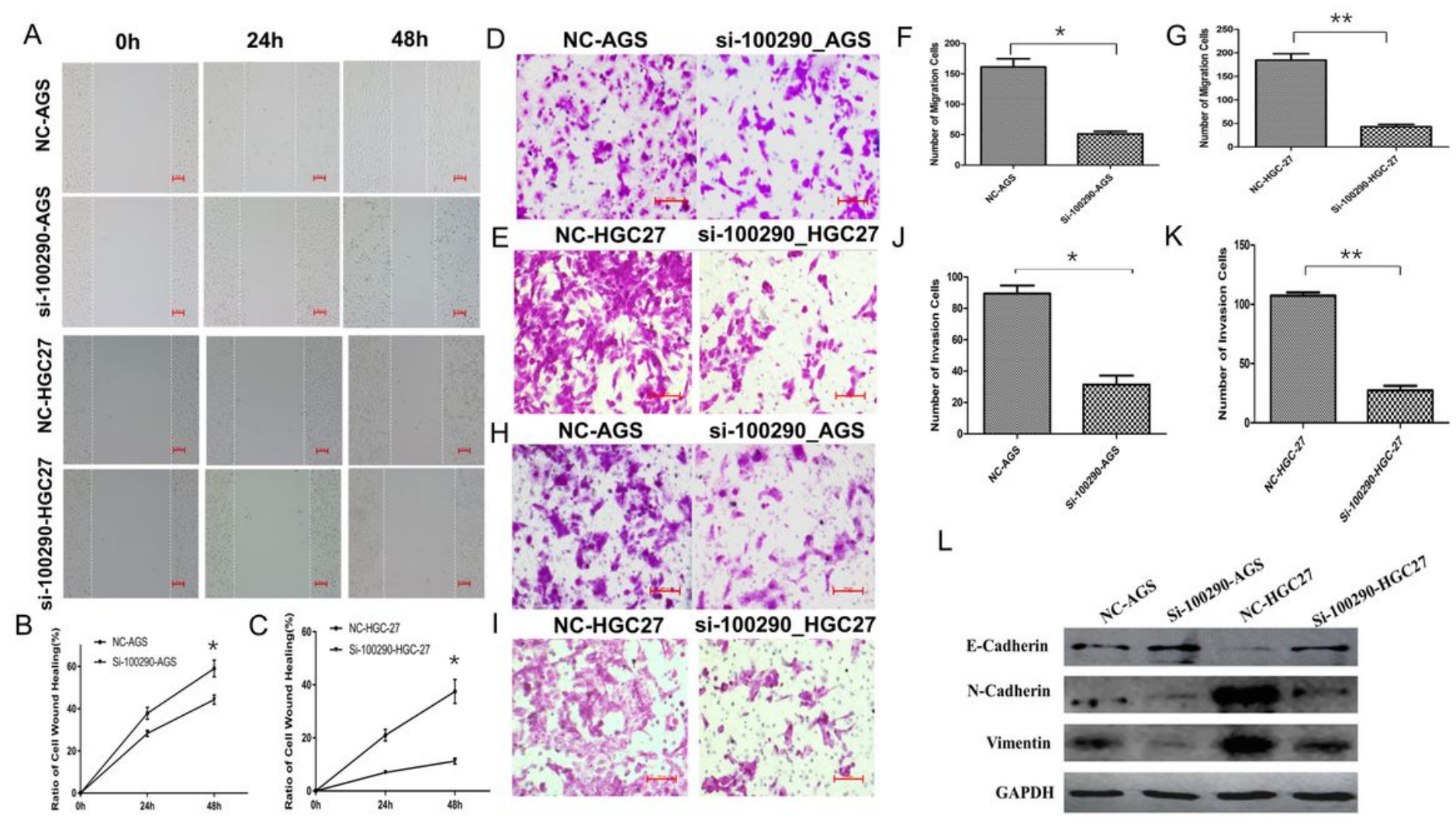

\section{Figure 4}

Decreased circRNA_100290 expression suppresses GC cell migration and invasion ability and impedes EMT. (A) Wound-healing assay was performed to evaluate the effects of circRNA_100290 on migration abilities of AGS and HGC27 cells at 0h, 24h, 48h. (B\&C) The statistical graphs showed that knockingdown circRNA_100290 obviously inhibited AGS and HGC27 cell wound healing ( $\left.{ }^{*} P<0.05\right)$. (D\&E) Transwell migration assay was performed to evaluate the effects of circRNA_100290 on migration abilities of AGS and HGC27 cells. (F\&G) The statistical graphs showed that decreased circRNA_100290 expression induced the less AGS and HGC27 cells migrated into lower chamber compared with control groups ( $* P<0.05, * * P<0.01)$. (H\&l) Transwell invasion assay was performed to evaluate the effects of circRNA_100290 on invasion abilities of AGS and HGC27 cells. (J\&K) The statistical graphs displayed that reduced circRNA_100290 expression mediated less AGS and HGC27 cells invaded into lower chamber compared with control groups $\left({ }^{*} P<0.05, * \star P<0.01\right)$. (L) Western blot assay was preformed to evaluate the expression of EMT-related proteins including E-cadherin, N-cadherin and Vimentin. 


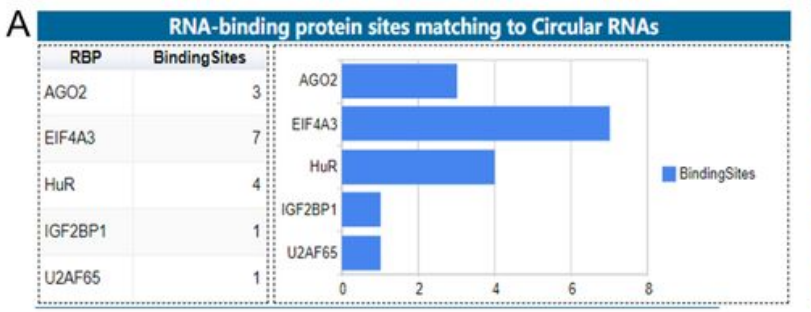

\begin{tabular}{|c|c|}
\hline \multicolumn{2}{|c|}{ RNA-binding protein sites matching flanking regions of Circular RNA } \\
\hline RNA-binding Protein & \# Tags \\
\hline$\underline{\text { EFAA3 } 3}$ & 3 \\
\hline$\underline{\text { HUR }}$ & 1 \\
\hline$\underline{\text { GF2BP2 }}$ & 1 \\
\hline$\underline{\text { PTB }}$ & 1 \\
\hline$\underline{\text { ULAF65 }}$ & 2 \\
\hline \hline
\end{tabular}

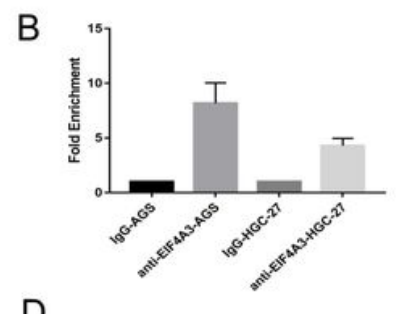

C

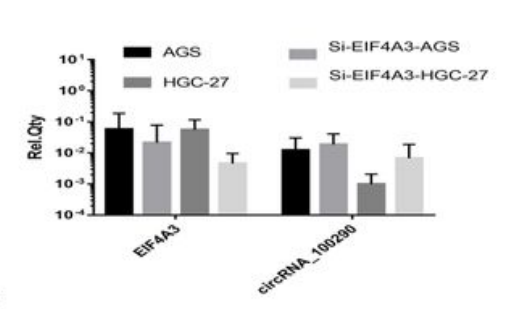

D

$\mathrm{E}$

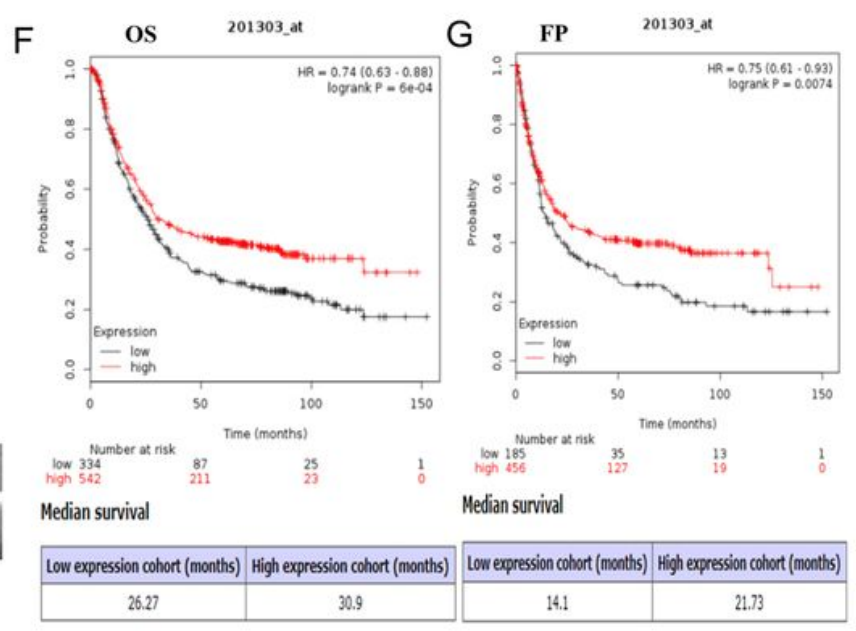

Figure 5

Transcription regulation of EIF4A3 on circRNA_100290 in GC. (A) Prediction of circRNA_100290 related RBPs by using Circinteractome software. (B) RIP assay was performed to determine fold enrichment of flanking region of circRNA_100290 by EIF4A3. (C) Detection of circRNA_100290 by qRT-PCR after knocking down of EIF4A3 in AGS and HGC-27 cells. (D) Detection of EIF4A3 expression in $31 \mathrm{GC}$ tissues and paired normal tissues was conducted by qRT-PCR assay. Decreased expression of EIF4A3 was observed in GC tissues. Data are means \pm SEM. ${ }^{\star} * P<0.01$. (E) Western blot assay was performed to determine EIF4A3 expression in $31 \mathrm{GC}$ tissues and paired normal tissues. (F\&G) Prognosis analysis about OS and FP of ITGA11 in GC patients from the Kaplan-Meier plotter website. 